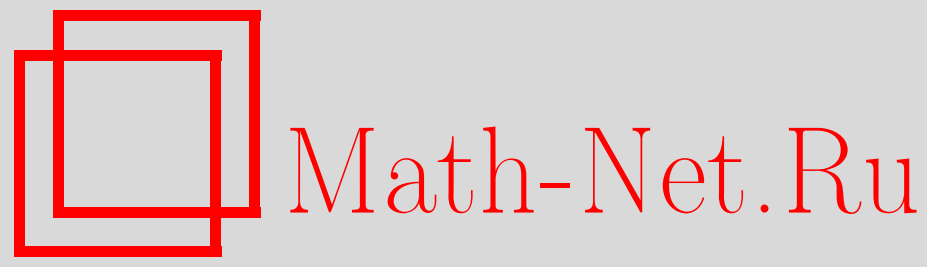

М. С. Агранович, K теории задач Дирихле и Неймана для линейных сильно эллиптических систем в липшицевых областях, Функи. анализ и его прил., 2007, том 41, выпуск 4, 1-21

DOI: https://doi.org/10.4213/faa2875

Использование Общероссийского математического портала MathNet.Ru подразумевает, что вы прочитали и согласны с пользовательским соглашением http://www.mathnet.ru/rus/agreement

Параметры загрузки:

IP : 54.198 .187 .58

26 апреля 2023 г., 03:55:44

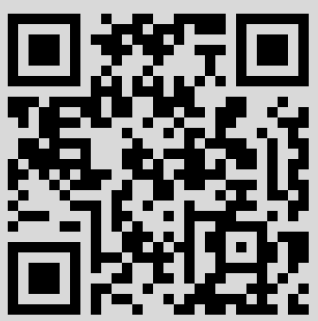




\title{
К теории задач Дирихле и Неймана для линейных сильно эллиптических систем в липшицевых областях ${ }^{*}$
}

\author{
(c) 2007. М. С. АГРАнович
}

\section{§1. Содержание работы}

В этой статье рассматриваются задачи Дирихле и Неймана в ограниченной липшицевой области $\Omega \subset \mathbb{R}^{n}, n \geqslant 2$, для сильно эллиптических систем со структурой Дуглиса-Ниренберга. Исходная постановка задач - вариационная, или слабая, т. е. классическая, с использованием $L_{2}$-пространств СоболеваСлободецкого $H^{\sigma}(\Omega)=H_{2}^{\sigma}(\Omega)$, см. $\S 2$. Но речь идет о регулярности решений в терминах их принадлежности (и принадлежности правых частей) к пространствам потенциалов $H_{p}^{\sigma}(\Omega)$ или пространствам Бесова $B_{p}^{\sigma}(\Omega)$. Предположения о гладкости коэффициентов в известной мере минимизированы. Как и в статье автора [3], рассмотрения основаны на методе исследования регулярности, предложенном Саваре в [23] для случая $p=2$, см. $\S 3$, и на средствах теории интерполяции. Вариационная постановка при этом, как и в [3], обобщается на пространства $H_{p}^{\sigma}(\Omega)$, а также на пространства $B_{p}^{\sigma}(\Omega)$.

Для уравнений высокого порядка в липшицевой области непростым является вопрос о смысле граничных условий. В основной части нашей статьи они предполагаются однородными и легко описываются выбором пространств. В задаче Дирихле с неоднородными граничными условиями мы предполагаем наличие функции, которая является носителем данных Дирихле. Такая точка зрения общеизвестна. В п. 5.1 мы показываем, что она согласуется с заданием этих данных в пространстве так называемых наборов Уитни, ср. [28] и [1]. Кое-что мы сумеем сказать и о задаче с неоднородными условиями Неймана в п. 5.2.

В [3] рассмотрены сильно эллиптические системы 2-го порядка в предположении их формальной самосопряженности. В настоящей статье рассматриваются системы Дуглиса-Ниренберга произвольных порядков, и в задаче Дирихле требуется положительная определенность действительной части главного символа, а в задаче Неймана - коэрцитивность соответствующей интегральной формы на простейшем пространстве, в котором можно искать вариационное решение. При этом на структуру формы накладывается дополнительное ограничение, см. ниже (2.11). Оно на самом деле необходимо для использования метода Саваре и в рассмотренном в [3] случае. Наиболее существенное продвижение по сравнению с [3] состоит в том, что на плоскости параметров $s$ и $t=1 / p$ однозначная разрешимость получена в прямоугольнике, боковые стороны которого находятся на сторонах единичного квадрата $Q$ (см. обозначения в [3] и ниже в $\S 4$ ). Это показано в $\S 4$ при помощи оценки из [25]. В [3] результаты были получены в

* Работа выполнена при поддержке Российского фонда фундаментальных исследований, грант №07-01-00287-a. 
пространствах $H_{p}^{\sigma}(\Omega)$ для прямоугольника, лежащего строго внутри $Q$. Усилив их, мы в известной мере, в рамках рассматриваемых здесь задач, «догоняем» работы «невариационного» направления, см. следующий абзац, и получаем новые результаты для систем в случае задачи Неймана. В п. 5.5 мы указываем аналоги наших результатов для тех же задач в пространствах Бесова $B_{p}^{\sigma}(\Omega)$ вместо пространства потенциалов $H_{p}^{\sigma}(\Omega)$, ср. [11], [10], [15] и [1].

Рассмотрения в [23], [2], [3], более ранней работе Костабеля [6] и в настоящей статье являются альтернативой к глубокому «невариационному» подходу к исследованию граничных задач в липшицевых областях. Соответствующая литература чрезвычайно обширна в случае уравнений и систем 2-го порядка, см., в частности, ссылки в [3] и в названных там работах. Но и уравнениям и системам высоких порядков посвящено немало работ, назовем статьи [8], [28], [20], [29], [7], [1], [30], [24], [14], в которых можно найти дальнейшие ссылки. Мы кратко сравним наши результаты с результатами этих работ в 6 . Пока отметим только, что при наших предположениях и нашем подходе задача Неймана не сложнее, чем задача Дирихле.

Автор искренне благодарен Р. В. Дудучаве, просмотревшему рукопись и сделавшему полезные замечания.

\section{§2. Постановка вариационных задач}

Система Дуглиса-Ниренберга - довольно громоздкий объект, но мы введем обозначения так, чтобы читатель мог при желании проследить все построения на случае скалярного уравнения.

Пусть $\Omega$ - ограниченная область в $\mathbb{R}^{n}$ с липшицевой границей Г. Предположим, что в $\Omega$ задана система уравнений в частных производных

$$
L u(x)=L_{0} u(x)+\cdots+\tau u(x)=f(x) .
$$

Здесь $u(x)=\left(u_{1}(x), \ldots, u_{l}(x)\right)^{\prime}$ и $f(x)=\left(f_{1}(x), \ldots, f_{l}(x)\right)^{\prime}$ - вектор-функции, столбцы высоты $l$. Соответственно $L$ и старшая часть $L_{0}$ этого оператора матричные операторы с размерами матриц $l \times l$. Мы предположим, что задана система целых положительных чисел $m_{1}, \ldots, m_{l}$ и что элементы с индексами $j, k$ этих матриц имеют порядки не выше $m_{j}+m_{k}$. Старшие части $L_{j, k}$ этих скалярных операторов записываются в дивергентной форме:

$$
L_{j, k}(x, D)=\sum_{\substack{|\alpha|=m_{j},|\beta|=m_{k}}} D^{\alpha}\left[a_{\alpha, \beta}^{j, k}(x) D^{\beta} \cdot\right] .
$$

Коэффициенты $a_{\alpha, \beta}^{j, k}(x)$, вообще говоря, комплекснозначны, и формальная самосопряженность оператора (2.2) не предполагается. Минимальное предположение о регулярности коэффициентов состоит в том, что они непрерывны в $\bar{\Omega}$. Этого достаточно при выводе неравенства Гординга [18]. Начиная с §3, мъь предполагаем их удовлетворяющими равномерному условию Липшииа. Как обычно, $D^{\alpha}=D_{1}^{\alpha_{1}} \cdots D_{n}^{\alpha_{n}}$, где $D_{j}=-i \partial / \partial x_{j}$. Порядок дифференцирования в этих смешанных производных безразличен, так как они применяются к функциям из пространств, в которых плотны гладкие функции. Числовой параметр $\tau$ имеет неотрицательную действительную часть. Мы не выписываем обозначенные многоточием остальные младшие члены и до n. 5.3 nредnолагаем, что они отсутствуют. Главный символ системы есть матрица $L_{0}(x, \xi)$ с элементами $L_{j, k}(x, \xi)$. 
Он подчиняется условию сильной эллиптичности - условию равномерной положительной определенности его действительной части при вещественных $\xi$ :

$$
\operatorname{Re} L_{0}(x, \xi) \geqslant C_{1} E(\xi),
$$

где $E(\xi)$ - диагональная матрица с элементами $|\xi|^{2 m_{j}}$ на главной диагонали $(j=1, \ldots, l)$ и $C_{1}$ - положительная постоянная. Как обычно, под действительной частью матрицы понимается ее полусумма с эрмитово сопряженной матрицей. Определение сильной эллиптичности было введено в работе Вишика [31] в случае совпадения чисел $m_{j}$. Общий случай рассмотрен в работе Ниренберга [18]. Сильно эллиптические системы со структурой Дуглиса-Ниренберга (т. е. с не одинаковыми $m_{j}$ ) встречаются в задачах для тонких упругих оболочек и пластин. См., например, [9], приложение 2, §2, и [16], §4. Там, правда, старшая часть системы распадается на матричный оператор Ламе с $m=1$ и скалярный бигармонический оператор с $m=2$.

Пусть $(u, v)_{\Omega}$ - стандартное скалярное произведение в пространстве $L_{2}(\Omega)$ вектор-функций размерности $l$. Предположив на минуту $u$ и $v$ финитными бесконечно гладкими функциями с носителями, лежащими внутри $\Omega$, интегрированием по частям выражения $\left(L_{0}(x, D) u, v\right)_{\Omega}$ получим полуторалинейную форму

$$
\Phi_{0}(u, v)=\int_{\Omega} \sum_{j, k=1}^{l} \sum_{\substack{\alpha\left|=m_{j},\\\right| \beta \mid=m_{k}}} a_{\alpha, \beta}^{j, k}(x) D^{\beta} u_{k}(x) \overline{D^{\alpha} v_{j}(x)} d x .
$$

Положим

$$
\Phi_{\tau}(u, v)=\Phi_{0}(u, v)+\tau(u, v)_{\Omega}, \quad \Phi_{\tau}(u)=\Phi_{\tau}(u, u) \quad(\tau \geqslant 0) .
$$

До §5 граничные условия в задачах Дирихле и Неймана предполагаются однородными. Вариационные постановки этих задач состоят в том, что решение $u$ должно удовлетворять вариационному тождеству

$$
(f, v)_{\Omega}=\Phi_{\tau}(u, v)
$$

при произвольной пробной функции $v$. Это просто формула Грина без граничных членов. Но здесь нужно указать функциональные пространства для $u, v$ и $f$.

Сначала нам будут нужны простейшие пространства скалярных функций. Пусть $\sigma$ - вещественное число. Через $H^{\sigma}\left(\mathbb{R}^{n}\right)$ обозначается $L_{2}$-пространство Соболева-Слободецкого порядка $\sigma$ в $\mathbb{R}^{n}$ (см. начало $\S 4$ при $p=2$ ). Через $H^{\sigma}(\Omega)$ - пространство сужений функций из $H^{\sigma}=H^{\sigma}\left(\mathbb{R}^{n}\right)$ (обобщенных функций при $\sigma<0)$ на $\Omega$ с нормой, равной нижней грани норм в $H^{\sigma}$ всех функций с данным сужением. Через $\widetilde{H}^{\sigma}(\Omega)$ - подпространство функций в $H^{\sigma}$ с носителями в $\bar{\Omega}$ и нормой, которая берется из $H^{\sigma}$. В $H^{\sigma}(\Omega)$ плотны функции, гладкие в $\bar{\Omega}$, а в $\widetilde{H}^{\sigma}(\Omega)$ - гладкие функции с носителями в $\Omega$, продолженные нулем вне $\Omega$.

При $\sigma>-1 / 2, \sigma-[\sigma] \neq 1 / 2([\sigma]$ - целая часть числа $\sigma)$ пространство $\widetilde{H}^{\sigma}(\Omega)$ можно отождествить с пополнением $\stackrel{\circ}{H^{\sigma}}(\Omega)$ линеала $C_{0}^{\infty}(\Omega)$ в $H^{\sigma}(\Omega)$, нормы эквивалентны.

Пространства $H^{\sigma}(\Omega)$ и $\widetilde{H}^{-\sigma}(\Omega)$ взаимно сопряжены относительно продолжения скалярного произведения в $L_{2}(\Omega)$ с плотных в них гладких функций на 
прямое произведение этих пространств. См. ниже формулу (4.3) при $p=p^{\prime}=2$, а также пояснения в [3], §3, и [4].

Теперь введем пространства вектор-функций

$\mathscr{H}^{ \pm \mathbf{m}}(\Omega)=H^{ \pm m_{1}}(\Omega) \times \cdots \times H^{ \pm m_{l}}(\Omega), \quad \widetilde{\mathscr{H}}^{ \pm \mathbf{m}}(\Omega)=\widetilde{H}^{ \pm m_{1}}(\Omega) \times \cdots \times \widetilde{H}^{ \pm m_{l}}(\Omega)$.

Нормы вектор-функций в этих пространствах определяются как квадратные корни из суммы квадратов норм их компонент. В задаче Дирихле

$$
u, v \in \widetilde{\mathscr{H}}^{\mathbf{m}}(\Omega), \quad f \in \mathscr{H}^{-\mathbf{m}}(\Omega),
$$

и здесь заложено равенство нулю данных Дирихле у $u$ и $v$. В задаче Неймана

$$
u, v \in \mathscr{H}^{\mathbf{m}}(\Omega), \quad f \in \widetilde{\mathscr{H}}^{-\mathbf{m}}(\Omega) .
$$

В обоих случаях пространства для $v$ и для $f$ взаимно сопряжены относительно продолжения на их прямое произведение скалярного произведения в

$$
\mathscr{L}_{2}(\Omega)=L_{2}(\Omega) \times \cdots \times L_{2}(\Omega)
$$

с гладких вектор-функций.

Напомним неравенство Гординга в следующей форме: если система сильно эллиптична и $\operatorname{Re} \tau$ достаточно велико, то для функций $v$ из $\widetilde{\mathscr{H}}^{\mathbf{m}}(\Omega)$

$$
\operatorname{Re} \Phi_{\tau}(v) \geqslant C_{2}\|v\|_{\mathscr{H}^{\mathbf{m}}(\Omega)}^{2}
$$

с положительной постоянной $C_{2}$ (см. [18]). Неравенство в обратную сторону очевидно, так что квадратный корень из $\operatorname{Re} \Phi_{\tau}(v)$ оказывается нормой, эквивалентной обычной норме в этом пространстве. Из (2.9) следует однозначная разрешимость задачи Дирихле: единственность сразу получается из равенства нулю формы $\Phi_{\tau}(u)$ при $f=0$, а существование проверяется при помощи известной леммы Вишика [31]-Лакса-Мильграма [13].

В случае задачи Неймана мы, как это принято, предположим выполненным аналогичное неравенство для функций из $\mathscr{H}^{\mathbf{m}}(\Omega)$ при достаточно большом $\operatorname{Re} \tau$ :

$$
\operatorname{Re} \Phi_{\tau}(v) \geqslant C_{2}\|v\|_{\mathscr{H}^{\mathbf{m}}(\Omega)}^{2} .
$$

Будем говорить, что это условие коэриитивности формы $\operatorname{Re} \Phi_{\tau}(v)$ на пространстве $\mathscr{H}^{\mathbf{m}}(\Omega)$. Известны достаточные условия; см., в частности, [17], гл. $3, \S 7$, а также [2], §1, и приведенные там ссылки. См. также примеры немного ниже. При условии коэрцитивности (2.10) квадратный корень из $\operatorname{Re} \Phi_{\tau}(v)$ - норма в $H^{\mathbf{m}}(\Omega)$, эквивалентная обычной, и задача Неймана оказывается однозначно разрешимой.

Метод Саваре не является всеобъемлющим. Для исследования регулярности решений задач Дирихле и Неймана этим методом нам нужно наложить на систему дополнительное условие:

$$
F(x, \zeta):=\operatorname{Re} \sum_{j, k=1}^{l} \sum_{\substack{\alpha\left|=m_{j},\\\right| \beta \mid=m_{k}}} a_{\alpha, \beta}^{j, k}(x) \zeta_{\beta}^{k} \bar{\zeta}_{\alpha}^{j} \geqslant 0
$$

для любых чисел $\zeta_{\alpha}^{j}$ во всех точках $x \in \Omega$. Это условие равносильно неотрицательности выражения под знаком интеграла в формуле для $\Phi_{0}(u)$ в каждой точке на всех рассматриваемых функциях $u(x)$. 
Приведем примеры систем, для которых выполнены условие коэрцитивности и условие (2.11).

1. Скалярное сильно эллиптическое уравнение 2-го порядка.

2. Системы 2-го порядка с такой старшей частью $\sum D_{j} A_{j, k} D_{k}$, что действительная часть клеточной матрицы размеров $n l \times n l$ с клетками $A_{j, k}(x)$ равномерно положительно определена.

3. Системы в теории упругости, как изотропной (система Ламе), так и анизотропной, и более общие системы 2-го порядка, указанные в [2], §1, см. там условие II.

4. Уравнение с полигармоническим оператором $(-1)^{m} \Delta^{m} u$ в старшей части. Здесь мы имеем в виду возможность записать соответствующую форму $\Phi_{0}(v)$ в виде

$$
\Phi_{0}(v)=\int_{\Omega} \sum_{|\alpha|=m} C_{\alpha}\left|D^{\alpha} v\right|^{2} d x,
$$

где $C_{\alpha}$ - положительные коэффициенты.

5. Система со старшей частью $\sum_{|\alpha|=m} D^{\alpha} A_{\alpha}(x) D^{\alpha} u(x)$, где действительные части матриц $A_{\alpha}(x)$ равномерно положительно определены в слагаемых с несмешанными производными $D^{\alpha}$ и неотрицательно определены в остальных слагаемых.

Здесь автор должен отметить, что в [3] сделана ссылка на вариант теоремы Саваре, изложенный в обзоре [2]. Но в [2] предполагалось выполненным уже упомянутое условие II, из которого (2.11) вытекает. В [3] следовало предположить выполненным условие (2.11); это не было сделано по недосмотру автора.

Всюду дальше оператор $L(x, D)=L_{0}(x, D)+\tau$ предполагается равномерно сильно эллиптическим и в случае задачи Неймана удовлетворяющим условию коэрцитивности формы $\operatorname{Re} \Phi_{\tau}(v)$ на $\mathscr{H}^{\mathbf{m}}(\Omega)$. Предполагается также выполненным условие (2.11). Всюду, кроме п. 5.3, в каждой из задач, Дирихле и Неймана, число $\tau$ считается зафиксированным так, что имеет место однозначная разрешимость.

\section{§3. Обобщение результатов Саваре}

Пусть $s$ - число из интервала $(-1 / 2,1 / 2)$. Через $\mathscr{H}^{ \pm \mathbf{m}+s}(\Omega)$ и $\widetilde{\mathscr{H}}^{ \pm \mathbf{m}}+s(\Omega)$ обозначим пространства, определяемые аналогично $\mathscr{H}^{ \pm \mathbf{m}}(\Omega)$ и $\widetilde{\mathscr{H}^{ \pm}} \mathbf{m}(\Omega)$, но с добавлением ко всем индексам $\pm m_{j}$ числа $s$. В следующей теореме речь идет о вариационном решении, первоначально определенном при $s=0$.

Теорема 1. Пусть $s \in(0,1 / 2)$.

1. Если $f \in \mathscr{H}^{-\mathbf{m}+s}(\Omega)$, то решение задачи Дирихле принадлежит $\widetilde{\mathscr{H}}^{\mathbf{m}+s}(\Omega)$.

2. Если $f \in \widetilde{\mathscr{H}}^{-\mathbf{m}+s}(\Omega)$, то решение задачи Неймана принадлежсит $\mathscr{H}^{\mathbf{m}+s}(\Omega)$.

Метод получения подобных результатов, основанный на модификации метода разностных отношений Ниренберга [18], разработан в [23]. Рассмотренные там уравнения, вообще говоря, нелинейны. Из линейных задач подробно рассмотрены задачи Дирихле и Неймана для скалярного уравнения 2-го порядка в дивергентной форме $\operatorname{div} a(x) \operatorname{grad} u(x)=f(x)$ с симметричной вещественной 
положительно определенной матрицей $a(x)$ и кратко - задача Дирихле для бигармонического уравнения. В обзоре [2] теорема 1 проверена для систем 2-го порядка с самосопряженной главной частью фактически при условии (2.11). Мы сейчас должны доказать эту теорему в полной общности. При этом будут использованы некоторые добавочные соображения. Кроме того, мы несколько уточним рассуждения, изложенные в [2].

Вводимые дальше обозначения похожи на обозначения в [23] и [2], но приспособлены к общему случаю. Мы, однако, не будем повторять некоторые очевидные детали, если будет удобно сослаться на [23] или [2]. Сначала мы подробно рассмотрим задачу Неймана. В конце доказательства будут кратко указаны изменения, нужные в случае задачи Дирихле.

В доказательстве понадобится пространство Бесова $B_{2, \infty}^{s}(\Omega)$ с нецелым $s>0$, пока это пространство скалярных функций. Пусть $d$ - малое положительное число. Обозначим через $\Omega_{d}$ множество точек области $\Omega$, отстоящих от ее границы больше чем на $d$. Пусть $\sigma \in(0,1)$ и $D-$ множество векторов, порождающее $\mathbb{R}^{n}$ и звездное относительно начала координат. Например, это может быть конус с вершиной в начале. Для функций $v$ в $\Omega$ определим полунорму $[v]_{\sigma, \Omega}$ равенством

$$
[v]_{\sigma, \Omega}^{2}=\sup _{h \in D \backslash 0,|h|<d}|h|^{-2 \sigma} \int_{\Omega_{|h|}}|v(x+h)-v(x)|^{2} d x .
$$

Норму в $B_{2, \infty}^{s}(\Omega)$ при нецелом $s>0$ можно определить равенством

$$
\|v\|_{B_{2, \infty}^{s}(\Omega)}=\|v\|_{H^{[s]}(\Omega)}+\sum_{|\alpha|=[s]}\left[D^{\alpha} v\right]_{s-[s], \Omega} .
$$

Пространство $B_{2, \infty}^{s}(\Omega)$ определяется как пополнение линеала $C^{\infty}(\bar{\Omega})$ по этой норме. Ср. [23], стр. 13.

В этом пространстве возможна следующая локализация. Пусть замыкание $\bar{\Omega}$ покрыто конечной системой открытых шаров $O_{j}(j=0, \ldots, N)$, и пусть $\sum_{j} \phi_{j} \equiv 1$ - разбиение единицы в окрестности множества $\bar{\Omega}$, состоящее из гладких функций $\phi_{j}$ с носителями в $O_{j}$. Тогда

$$
\|u\|_{B_{2, \infty}^{s}(\Omega)} \leqslant C^{\prime} \sum\left\|\phi_{j} v\right\|_{B_{2, \infty}^{s}\left(O_{j} \cap \Omega\right)} \leqslant C^{\prime \prime}\|u\|_{B_{2, \infty}^{s}(\Omega)},
$$

где $C^{\prime}, C^{\prime \prime}$ - постоянные. Это позволяет по-разному выбирать множества $D$ для разных шаров $O_{j}$, имеющих общие точки с границей.

Пространство $\mathscr{B}_{2, \infty}^{\mathbf{m}+1 / 2}(\Omega)$ вектор-функций $v=\left(v_{1}, \ldots, v_{l}\right)^{\prime}$ определим как прямое произведение пространств $B_{2, \infty}^{m_{j}+1 / 2}(\Omega)(j=1, \ldots, l)$ с нормой, равной сумме норм компонент в этих пространствах.

Пусть $u \in \mathscr{H}^{\mathbf{m}}(\Omega)$ - решение задачи Неймана, см. (2.6) и (2.8).

Лемма (ср. [23], стр. 22, в случае $\Delta^{2}$ ). Пусть правая часть $f$ системы принадлежит пространству $\widetilde{\mathscr{H}}^{-\mathbf{m}+1}(\Omega)$. Тогда решение $u(x)$ принадлежнт пространству Бесова $\mathscr{B}_{2, \infty}^{\mathbf{m}+1 / 2}(\Omega)$ и справедливо неравенство

$$
\|u\|_{\mathscr{B}_{2, \infty}^{\mathbf{m}+1 / 2}(\Omega)}^{2} \leqslant C_{3}\|f\|_{\widetilde{\mathscr{H}}-\mathbf{m}+1(\Omega)}\|u\|_{\mathscr{H}^{\mathbf{m}}(\Omega)}
$$

с не зависящей от $u(x)$ постоянной $C_{3}$.

Из этой леммы теорема будет выведена при помощи интерполяции. 
Начнем с некоторой подготовки к доказательству леммы.

Положим

$$
\Psi(u)=\operatorname{Re}\left(\Phi_{\tau}(u)-2(f, u)_{\Omega}\right) .
$$

Эта форма принимает на решении наименьшее значение. Действительно, пусть $v$ тоже принадлежит $\mathscr{H}^{\mathbf{m}}(\Omega)$; тогда

$$
\Psi(u+v)-\Psi(u)=\operatorname{Re} \Phi_{\tau}(v)+2 \operatorname{Re}\left[\Phi_{\tau}(u, v)-(f, v)_{\Omega}\right] .
$$

Здесь выражение в квадратных скобках равно нулю, так что правая часть неотрицательна.

Более того, в силу неравенства $(2.10)$ на функциях из $\mathscr{H}^{\mathbf{m}}(\Omega)$

$$
C_{2}\|v\|_{\mathscr{H}^{\mathbf{m}}(\Omega)}^{2} \leqslant \Psi(u+v)-\Psi(u) .
$$

В частности, здесь оценивается квадрат $L_{2}$-нормы всех старших производных от $v$.

Липшицева поверхность, как известно, обладает равномерным свойством конуса. Каждая точка $x_{0}$ на $Г$ является вершиной двух прямых круговых конусов $C^{+}\left(x_{0}\right)$ и $C^{-}\left(x_{0}\right)$, конгруэнтных фиксированному конусу и лежащих соответственно внутри $\Omega$ и внутри дополнения к $\Omega$, кроме вершины $x_{0}$. Локально оси этих конусов можно выбирать параллельными. При рассмотрении задачи Неймана понадобятся конусы $C^{+}$. Пусть $r-$ высота этих конусов.

Через $D=D\left(x_{0}\right)$ обозначим множество таких векторов $h \in \mathbb{R}^{n}$, выходящих из начала, что $x_{0}+h \in C^{+}\left(x_{0}\right)$. Через $\Omega_{\rho}(x)$ обозначим пересечение области $\Omega$ с шаром $O_{\rho}\left(x_{0}\right)$ радиуса $\rho$ с центром в $x_{0}$. Далее понадобятся также $O_{2 \rho}\left(x_{0}\right)$ и $O_{3 \rho}\left(x_{0}\right)$. Направление оси конуса $C^{+}\left(x_{0}\right)$ и числа $\rho \in(0, r)$ и $d\left(x_{0}\right) \in(0, \rho)$ зафиксируем так, что

$$
x \in O_{2 \rho}\left(x_{0}\right), \quad h \in D\left(x_{0}\right), \quad|h| \leqslant d\left(x_{0}\right) \Longrightarrow x+h \in O_{3 \rho}\left(x_{0}\right) .
$$

Эти числа, $\rho$ и $d$, можно считать не зависящими от $x_{0}$.

Пусть $\varphi(x)$ - бесконечно гладкая вещественная скалярная функция со значениями на $[0,1]$, равная 1 в $O_{\rho}\left(x_{0}\right)$ и нулю вне $O_{2 \rho}\left(x_{0}\right)$. Эти функции можно предположить сдвигами $\psi\left(x-x_{0}\right)$ фиксированной функции $\psi(x)$.

Теперь положим

$$
\mathscr{T}_{h} v(x)=\varphi(x) v(x+h)+[1-\varphi(x)] v(x) .
$$

Эта вектор-функция определена в $\Omega$ и принадлежит пространству $\mathscr{H}^{\mathbf{m}+s}(\Omega)$ вместе с $v$. Через $\zeta(x)$ обозначим вектор-функцию с компонентами $\zeta_{j}^{\alpha}(x),|\alpha|=$ $m_{j}, j=1, \ldots, l$. Если, в частности, это $D^{\alpha} v_{j}(x)$, то будем писать $\zeta(x)=\nabla^{\mathbf{m}} v(x)$. Положим также

$$
\mathscr{T}_{h} \zeta(x)=\varphi(x) \zeta(x+h)+[1-\varphi(x)] \zeta(x) .
$$

Здесь везде $h \in D\left(x_{0}\right)$ и $|h| \leqslant d$.

Квадратичная форма $F(x, \zeta)$ (см. $(2.11))$ при фиксированном $x$ отвечает эрмитовой полуторалинейной форме

$$
G(\zeta, \varsigma)=\operatorname{Re} \sum_{j, k=1}^{l} \sum_{\substack{\alpha\left|=m_{j},\\\right| \beta \mid=m_{k}}} a_{\alpha, \beta}^{j, k}(x) \zeta_{k}^{\beta} \bar{\varsigma}_{j}^{\alpha} .
$$


Квадратичная форма $G(\zeta)=G(\zeta, \zeta)=F(x, \zeta)$ выпукла: при любом числе $\varphi \in(0,1)$

$$
G(\varphi \zeta+(1-\varphi) \varsigma) \leqslant \varphi G(\zeta)+(1-\varphi) G(\varsigma) .
$$

Действительно, выпуклость квадратичной формы равносильна ее неотрицательности (см. [21], §4), а у нас последняя предположена в (2.11).

Доказательство леммы. Докажем следующую оценку:

$$
\Psi\left(\mathscr{T}_{h} v\right)-\Psi(v) \leqslant C_{4}|h|\|v\|_{\mathscr{H}^{\mathbf{m}\left(\Omega_{3 \rho}\right)}}\left(\|v\|_{\mathscr{H}^{\mathbf{m}}\left(\Omega_{3 \rho}\right)}+\|f\|_{\widetilde{\mathscr{C}}^{-\mathbf{m}+1}\left(\Omega_{3 \rho}\right)}\right) .
$$

Поясним, что если $v=u$ - решение, то левая часть неотрицательна и мажорирует величину $\operatorname{Re} \Phi_{\tau}(\varphi(x)(u(x+h)-u(x))$ (см. (3.6) и (3.9)), а значит, и квадрат нормы $\|\varphi(x)(u(x+h)-u(x))\|_{\mathscr{H}^{\mathbf{m}}\left(\Omega_{2 \rho}\right)}-$ см. (2.10).

Оценку вида (3.13) достаточно получить для слагаемых, из которых состоит форма $\Psi(v)$. Рассмотрим форму $\operatorname{Re} \Phi_{0}(v)$.

Сначала отметим, что если $\operatorname{supp} \zeta(x), \operatorname{supp} \varsigma(x) \subset \Omega_{2 \rho}$, то

$$
\int_{\Omega}[F(x, \varsigma(x)+\zeta(x))-F(x, \varsigma(x))] d x \leqslant C_{5}\|\zeta\|_{L_{2}\left(\Omega_{2 \rho}\right)}\left(\|\zeta\|_{L_{2}\left(\Omega_{2 \rho}\right)}+\|\varsigma\|_{L_{2}\left(\Omega_{2 \rho}\right)}\right)
$$

в силу неравенства Шварца. Здесь мы воспользовались тем, что $F(x, \zeta)$ - квадратичная форма по $\zeta$.

Далее, в силу только что отмеченной выпуклости формы $F$

$$
\begin{aligned}
F\left(x, \mathscr{T}_{h} \zeta(x)\right)-F(x, \zeta(x)) & \leqslant \varphi(x) F(x, \zeta(x+h)+(1-\varphi(x)) F(x, \zeta(x))-F(x, \zeta(x)) \\
& =\varphi(x)[F(x, \zeta(x+h))-F(x, \zeta(x)] .
\end{aligned}
$$

Поэтому

$$
\begin{aligned}
\int_{\Omega}\left[F\left(x, T_{h} \zeta(x)\right)-F(x, \zeta(x))\right] d x & \\
\leqslant & \int_{\Omega_{2 \rho}} \varphi(x)[F(x, \zeta(x+h))-F(x, \zeta(x))] d x \\
= & \int_{\Omega_{2 \rho}+h} \varphi(x-h)[F(x-h, \zeta(x))-F(x, \zeta(x))] d x \\
& \quad+\int_{\Omega_{2 \rho}+h} \varphi(x-h) F(x, \zeta(x)) d x-\int_{\Omega_{2 \rho}} \varphi(x) F(x, \zeta(x)) d x \\
= & \int_{\Omega_{3 \rho}} \varphi(x-h)[F(x-h, \zeta(x))-F(x, \zeta(x))] d x \\
\quad & \quad \int_{\Omega_{3 \rho}}[\varphi(x-h)-\varphi(x)] F(x, \zeta(x)) d x \\
\leqslant & C_{6}|h|\|\zeta\|_{L_{2}\left(\Omega_{3 \rho}\right)}^{2}
\end{aligned}
$$

с учетом того, что коэффициенты в $F(x, \zeta)$ и функция $\varphi(x)$ удовлетворяют условию Липшица. В силу формулы Лейбница

$$
D^{\alpha} \mathscr{T}_{h} v_{j}=\mathscr{T}_{h} D^{\alpha} v_{j}+\Sigma,
$$

где

$$
\Sigma=\sum_{\gamma+\delta=\alpha, \gamma \neq 0} C_{\gamma, \delta} D^{\gamma} \varphi(x) D^{\delta}\left[v_{j}(x+h)-v_{j}(x)\right] .
$$


Поэтому

$$
\begin{aligned}
& \operatorname{Re} \Phi_{0}\left(\mathscr{T}_{h} v\right)-\operatorname{Re} \Phi_{0}(v)=\int_{\Omega} {\left[F\left(x, \mathscr{T}_{h} \nabla^{\mathbf{m}} v+\Sigma\right)-F\left(x, \mathscr{T}_{h} \nabla^{\mathbf{m}} v\right)\right] d x } \\
&+\int_{\Omega}\left[F\left(x, \mathscr{T}_{h} \nabla^{\mathbf{m}} v\right)-F\left(x, \nabla^{\mathbf{m}} v\right)\right] d x
\end{aligned}
$$

Здесь второе слагаемое справа оценивается при помощи (3.16). Первое - при помощи (3.14) и неравенств типа

$$
\left\|D^{\delta} v_{j}(x+h)-D^{\delta} v_{j}(x)\right\|_{L_{2}\left(\Omega_{2 \rho}\right)} \leqslant|h|\left\|D^{\delta} v_{j}\right\|_{H^{1}\left(\Omega_{3 \rho}\right)},
$$

вытекающих из формулы Адамара для приращения функции, ср. [2], п. 2.4, стр. 27. Здесь $|\delta|<m_{j}$. Поэтому получаем

$$
\Phi_{0}\left(\mathscr{T}_{h} v\right)-\Phi_{0}(v) \leqslant C_{7}|h|\|v\|_{\mathscr{H}^{\mathbf{m}}\left(\Omega_{3 \rho}\right)}^{2} .
$$

Для функционала $(v, v)_{\Omega}$ без труда получается оценка

$$
\left(\mathscr{T}_{h} v, \mathscr{T}_{h} v\right)_{\Omega}-(v, v)_{\Omega} \leqslant C_{8}|h|\|\nabla v\|_{\mathscr{L}_{2}\left(\Omega_{3 \rho}\right)}^{2} .
$$

См. [2], там же. Для функционала $(f, v)_{\Omega}$

$$
\left(f, \mathscr{T}_{h} v-v\right)_{\Omega} \leqslant C_{9}|h|\|f\|_{\widetilde{\mathscr{H}}^{-\mathbf{m}+1}\left(\Omega_{2 \rho}\right)}\|\nabla v\|_{\mathscr{H}^{\mathrm{m}-1}\left(\Omega_{3 \rho}\right)} .
$$

Из (3.21)-(3.23) получаем (3.13).

Отсюда следует, что

$$
\left[\nabla^{\mathbf{m}} u\right]_{1 / 2, \Omega_{\rho}}^{2} \leqslant C_{10}\|f\|_{\widetilde{\mathscr{H}}-\mathbf{m}+1\left(\Omega_{3 \rho}\right)}\|u\|_{\mathscr{H}^{\mathbf{m}}\left(\Omega_{3 \rho}\right)} .
$$

Конструкция операторов $\mathscr{T}_{h}$ и результат распространяются на внутренние точки области. Из покрытия ее замыкания окрестностями $O_{\rho}$ выбираем конечное подпокрытие. Используя (3.24) и оценки

$$
\|u\|_{\mathscr{H}^{\mathbf{m}(\Omega)}} \leqslant C_{11}\|f\|_{\widetilde{\mathscr{H}}^{-\mathbf{m}(\Omega)}} \leqslant C_{12}\|f\|_{\mathscr{H}^{-\mathbf{m}+1}(\Omega)},
$$

в итоге приходим к (3.4).

Доказательство теоремы 1 . Обозначим через $\mathscr{L}$ отвечающий задаче оператор, переводящий $u$ в функционал $f$ по формуле (2.6). Из (3.4) и левого неравенства в (3.25) следует, что

$$
\|u\|_{\mathscr{B}_{2, \infty}^{\mathbf{m}+1 / 2}(\Omega)} \leqslant C_{12}\|f\|_{\widetilde{\mathscr{H}}-\mathbf{m}+1(\Omega)}\|f\|_{\widetilde{\mathscr{H}}^{-\mathbf{m}}(\Omega)} .
$$

Отсюда в силу предложения 4.2 из [23] (его формулировка повторена в [2], п. 2.3, утверждение 1) следует, что оператор $\mathscr{L}^{-1}$ действует ограниченным образом как оператор

$$
\left(\widetilde{\mathscr{H}}^{-\mathbf{m}}(\Omega), \widetilde{\mathscr{H}}^{-\mathbf{m}+1}(\Omega)\right)_{1 / 2,1} \mapsto \mathscr{B}_{2, \infty}^{\mathbf{m}+1 / 2}(\Omega) .
$$

Здесь слева имеется в виду вещественная интерполяция, см. определения, например, в [5], гл. 3. Кроме того, $\mathscr{L}^{-1}-$ ограниченный оператор из $\widetilde{\mathscr{H}}^{-\mathbf{m}}(\Omega)$ в $\mathscr{H}^{\mathbf{m}}(\Omega)$. Значит, это ограниченный оператор

$$
\left(\widetilde{\mathscr{H}}^{-\mathbf{m}}(\Omega),\left(\widetilde{\mathscr{H}}^{-\mathbf{m}}(\Omega), \widetilde{\mathscr{H}}^{-\mathbf{m}+1}(\Omega)\right)_{1 / 2,1}\right)_{t, 2} \mapsto\left(\mathscr{H}^{\mathbf{m}}(\Omega), \mathscr{B}_{2, \infty}^{\mathbf{m}+1 / 2}(\Omega)\right)_{t, 2}
$$

при $t \in(0,1)$. Заметим теперь, что

$$
\mathscr{B}_{2, \infty}^{\mathbf{m}+1 / 2}(\Omega)=\left(\mathscr{H}^{\mathbf{m}}(\Omega), \mathscr{H}^{\mathbf{m}+1}(\Omega)\right)_{1 / 2, \infty}
$$


(вещественная интерполяция, ср. [5], п. 6.4). По теоремам о реитерации и дуальности получается, что пространство справа в $(3.28)$ есть $\mathscr{H}^{\mathbf{m}+t / 2}(\Omega)$, а слева $\widetilde{\mathscr{H}}^{-\mathbf{m}+t / 2}(\Omega)$. Cp. [23], стр. 13-14. Заменяя $t / 2$ на $s$, получаем утверждение теоремы для задачи Неймана.

В случае задачи Дирихле нужно сделать очевидную замену пространств $\mathscr{H}^{\mathbf{m}+s}(\Omega)$ на $\widetilde{\mathscr{H}}^{\mathbf{m}+s}(\Omega)$ и $\widetilde{\mathscr{H}}^{-\mathbf{m}+s}(\Omega)$ на $\mathscr{H}^{-\mathbf{m}+s}(\Omega)$. Функция $v(x)$ считается равной нулю вне $\Omega$. Сдвиги $h$ делаются не внутрь области $\Omega$, а внутрь ее дополнения, т. е. в конусы $C^{-}$. Делается это для того, чтобы функция $\mathscr{T}_{h} v(x)$ принадлежала пространствам $\widetilde{\mathscr{H}}^{\mathbf{m}+s}(\Omega)$ вместе с $v$. Cp. [23], стр. 8-11.

Из теоремы 1 выводится

Теорема 2. 1. При $|s|<1 / 2$ задача Дирихле с правой частью $f \in \mathscr{H}^{-\mathbf{m}+s}(\Omega)$ имеет одно и толъко одно решение в $\widetilde{\mathscr{H}}^{\mathbf{m}+s}(\Omega)$.

2. При $|s|<1 / 2$ задача Неймана с правой частью $f \in \widetilde{\mathscr{H}}^{-\mathbf{m}+s}(\Omega)$ имеет одно и только одно решение в $\mathscr{H}^{\mathbf{m}+s}(\Omega)$.

Поясним, что задачи снова определяются тождеством (2.6); но при этом в задаче Дирихле

$$
u \in \widetilde{\mathscr{H}}^{\mathbf{m}+s}(\Omega), \quad v \in \widetilde{\mathscr{H}}^{\mathbf{m}-s}(\Omega), \quad f \in \mathscr{H}^{-\mathbf{m}+s}(\Omega),
$$

а в задаче Неймана

$$
u \in \mathscr{H}^{\mathbf{m}+s}(\Omega), \quad v \in \mathscr{H}^{\mathbf{m}-s}(\Omega), \quad f \in \widetilde{\mathscr{H}}^{-\mathbf{m}+s}(\Omega) .
$$

Таким образом, $u$ и $v$ теперь находятся, вообще говоря, в разных пространствах, а $v$ и $f$ остаются в сопряженных пространствах. При $s \in(0,1 / 2)$ утверждение получается сразу из теоремы 1 , а при $s \in(-1 / 2,0)-$ с использованием сопряженности вариационных задач (для формально сопряженных систем), отвечающих числам $s$ и $-s$, ср. [3].

Отметим, что соответствие между $f$ и $u$ в теореме 2 является изоморфизмом, а в лемме - нет.

\section{§4. Те же задачи в $H_{p}^{\sigma}$-пространствах}

Напомним определение пространства потенциалов $H_{p}^{\sigma}=H_{p}^{\sigma}\left(\mathbb{R}^{n}\right)$ (его называют также пространством бесселевых потенциалов, или пространством Лебега, или пространством Лиувилля). Будем предполагать, что

$$
\sigma \in \mathbb{R}, \quad p \in(1, \infty), \quad p p^{\prime}=p+p^{\prime} .
$$

Норма в пространстве $H_{p}^{\sigma}$ определяется равенством

$$
\|u\|_{H_{p}^{\sigma}}=\left\|\Lambda_{\sigma} u\right\|_{L_{p}\left(\mathbb{R}^{n}\right)},
$$

где $\Lambda_{\sigma}=F^{-1}\left(1+|\xi|^{2}\right)^{\sigma / 2} F, F$ - преобразование Фурье в смысле обобщенных функций. Это пространство определяется как пополнение линеала $C_{0}^{\infty}\left(\mathbb{R}^{n}\right)$ по норме (4.2).

Пространство $H_{p}^{\sigma}(\Omega)$ определяется как состоящее из сужений $u$ на $\Omega$ функций $w$ из $H_{p}^{\sigma}$ (обобщенных при $\left.\sigma<0\right)$ с нормой $\|u\|_{H_{p}^{\sigma}(\Omega)}=\inf \|w\|_{H_{p}^{\sigma}}:\left.w\right|_{\Omega}=u$.

Через $\widetilde{H}_{p}^{\sigma}(\Omega)$ обозначается подпространство функций в $H_{p}^{\sigma}$ (обобщенных при $\sigma<0)$ с носителями в $\bar{\Omega}$. Норма в $\widetilde{H}_{p}^{\sigma}(\Omega)$ берется из $H_{p}^{\sigma}$. 
Линеал $C^{\infty}(\bar{\Omega})$ содержится и плотен во всех $H_{p}^{\sigma}(\Omega)$. Линеал $C_{0}^{\infty}(\Omega)$ содержится и плотен во всех $\widetilde{H}_{p}^{\sigma}(\Omega) \cdot{ }^{1)}$

Пространство $\widetilde{H}_{p}^{\sigma}(\Omega)$ при $\sigma=k+\tau, k \in \mathbb{Z}_{+},-1 / p^{\prime}<\tau<1 / p$ можно отождествить с $\stackrel{\circ}{H} \underset{p}{\sigma}(\Omega)$ - пополнением линеала $C_{0}^{\infty}(\Omega)$ в $H_{p}^{\sigma}(\Omega)$; нормы эквивалентны.

Пространства $\widetilde{H}_{p^{\prime}}^{-\sigma}(\Omega)$ и $H_{p}^{\sigma}(\Omega)$ сопряжены относительно продолжения формы $(f, v)_{0, \Omega}$ на их прямое произведение при всех $\sigma$. Это продолжение определяется равенством

$$
(f, v)_{0, \Omega}=\lim \left(f_{k}, v_{k}\right)_{0, \Omega}=(f, \mathscr{E} v)_{0, \mathbb{R}^{n}},
$$

где функции $f_{k} \in C_{0}^{\infty}(\Omega)$ аппроксимируют $f$ в первом пространстве, а функции $v_{k} \in C^{\infty}(\bar{\Omega})-$ функцию $v$ во втором. Через $\mathscr{E}$ мы обозначили универсальный оператор продолжения функций из пространств в области $\Omega$ в аналогичные пространства в $\mathbb{R}^{n}$ из [22]. См. [3] и [4].

Пространства $H_{p}^{\sigma}(\Omega)$ и $\widetilde{H}_{p}^{\sigma}(\Omega)$ можно отождествить при $-1 / p^{\prime}<\sigma<1 / p$.

При $p=2$ индекс $p$ опускается и получаются использованные выше $L_{2}$-пространства $H^{\sigma}(\Omega)$ и $\widetilde{H}^{\sigma}(\Omega)$.

Введем прямые произведения и сокращенные обозначения для них. Пусть $|s|<1 / 2$. Положим

$$
\begin{aligned}
& \mathscr{H}_{p}^{ \pm \mathbf{m}+1 / p-1 / 2+s}(\Omega)=\prod_{j=1}^{l} H_{p}^{ \pm m_{j}+1 / p-1 / 2+s}(\Omega)=\mathscr{H}( \pm \mathbf{m}, p, s), \\
& \widetilde{\mathscr{H}}_{p}^{ \pm \mathbf{m}+1 / p-1 / 2+s}(\Omega)=\prod_{j=1}^{l} \widetilde{H}_{p}^{ \pm m_{j}+1 / p-1 / 2+s}(\Omega)=\widetilde{\mathscr{H}}( \pm \mathbf{m}, p, s) .
\end{aligned}
$$

Нормы в этих пространствах определяются как суммы норм в пространствах под знаком произведения.

В случае задачи Дирихле

$$
u \in \widetilde{\mathscr{H}}(\mathbf{m}, p, s), v \in \widetilde{\mathscr{H}}\left(\mathbf{m}, p^{\prime},-s\right), \quad f \in \mathscr{H}(-\mathbf{m}, p, s) .
$$

В случае задачи Неймана

$$
u \in \mathscr{H}(\mathbf{m}, p, s), \quad v \in \mathscr{H}\left(\mathbf{m}, p^{\prime},-s\right), \quad f \in \widetilde{\mathscr{H}}(-\mathbf{m}, p, s) .
$$

Здесь снова $u$ и $v$ лежат, вообще говоря, в разных пространствах, $v$ и $f-$ в сопряженных пространствах. Определение вариационного решения остается прежним, см. (2.6), но с использованием этих пространств.

Введем следующие обозначения (ср. [3]): $t=1 / p, \delta \in(0,1 / 2)$,

$$
Q=\{(s, t):|s|<1 / 2,0<t<1\}, \quad Q_{\delta}=\{(s, t):|s|<1 / 2,|t-1 / 2|<\delta\} .
$$

Под однозначной разрешимостью задачи Дирихле или Неймана в точке $(s, t)$ $\in Q$ будем понимать ее однозначную разрешимость в вариационной постановке в указанных выше пространствах с этими $s$ и $t$.

1) Здесь и дальше в аналогичных местах подразумевается, что функции из $C_{0}^{\infty}(\Omega)$ продолжаются нулем вне $\Omega$. 
Теорема 3. Пусть задана липшичева область $\Omega$. При сделанных выше предположениях найдется такое $\delta$, что задача Неймана однозначно разрешима $в$ точках $(s, t) \in Q_{\delta}$. Такое же утверждение верно для задачи Дирихле.

Здесь снова соответствия между $f$ и $u$ - изоморфизмы соответствующих пространств.

Доказательство теоремы 3. При комплексной интерполяции пространств $\mathscr{H}( \pm \mathbf{m}, p, s)$ вдоль любого отрезка, лежащего в $Q$, получаются те же пространства в соответствующих точках, и то же верно для пространств $\widetilde{\mathscr{H}}( \pm \mathbf{m}, p, s)$. См. [3] в случае $m_{j} \equiv 1$. Нам нужны вертикальные отрезки фиксированной длины чуть меньше 1 с серединами в точках с $t=1 / 2,|s|<1 / 2$. В этих точках оператор $\mathscr{L}(s, t): u \mapsto f$ обратим. Отсюда следует его обратимость на вертикальных отрезках длины $2 \delta$ с некоторым $\delta>0$ и теми же серединами в силу результата Шнейберга [25]. Более того, это $\delta$ можно считать не зависящим от абсциссы $s$. Это следует из ключевой для нас оценки для $\delta$ в [25] с учетом следующих двух обстоятельств. Во-первых, при рассматриваемых $(s, t)$ норма отвечающего задаче оператора $\mathscr{L}(s, t)$ равномерно ограничена. Во-вторых, норма обратного оператора $\mathscr{L}^{-1}(s, t)$ равномерно ограничена при $t=1 / 2$.

Приведем пояснения.

Пусть $\left\{E_{\alpha}\right\}$ и $\left\{F_{\alpha}\right\}, 0 \leqslant \alpha \leqslant 1$, - две шкалы банаховых пространств, полученные комплексной интерполяцией из интерполяционных пар $\left(E_{0}, E_{1}\right)$ и $\left(F_{0}, F_{1}\right)$. Пусть пересечение $E_{0} \cap E_{1}$ плотно в $E_{0}$ и $E_{1}$ и пересечение $F_{0} \cap F_{1}$ плотно в $F_{0}$ и $F_{1}$. Предположим, что имеются линейные ограниченные операторы $L_{j}$ из $E_{j}$ в $F_{j}(j=0,1)$, причем на $E_{0} \cap E_{1}$ они совпадают. Тогда определены единый линейный ограниченный оператор из $E_{0}+E_{1}$ в $F_{0}+F_{1}$ и его сужения $L_{\alpha}: E_{\alpha} \rightarrow F_{\alpha}$, причем последние являются линейными ограниченными операторами. См., например, [5], гл. 4.

Нужный нам результат Шнейберга можно сформулировать следующим образом. Пусть $M=\max \left\|L_{j}\right\|, j=0,1$, и пусть $L_{1 / 2}-$ изоморфизм. Тогда $L_{\alpha}-$ изоморфизм при

$$
\left|\alpha-\frac{1}{2}\right|<\frac{2}{\pi} \operatorname{arctg} \frac{1}{M\left\|L_{1 / 2}^{-1}\right\|} .
$$

Равномерная ограниченность операторов $\mathscr{L}(s, t)$ видна из формулы (2.6). Например, в случае задачи Неймана

$$
\left|\Phi_{\tau}(u, v)\right| \leqslant C\|u\|_{\mathscr{H}(\mathbf{m}, p, s)}\|v\|_{\mathscr{H}\left(\mathbf{m}, p^{\prime},-s\right)}
$$

с не зависящей от $(s, t)$ постоянной $C$. Действительно, рассмотрим оператор $a(x) D^{\alpha}$, где $|\alpha|=m$ и $a(x)$ - липшицева функция, как действующий из $H_{p}^{m+\sigma}(\Omega)$ в $H_{p}^{\sigma}(\Omega)$. Здесь у нас $p$ отделено от 1 и $\infty$ и поэтому $|\sigma| \leqslant \eta<1$. Как легко видеть, этот оператор равномерно ограничен. Следовательно,

$$
\|\mathscr{L}(x, t) u\|_{\widetilde{\mathscr{H}(-\mathbf{m}, p, s)}}=\sup \frac{\left|\Phi_{\tau}(u, v)\right|}{\|v\|_{\mathscr{H}\left(\mathbf{m}, p^{\prime},-s\right)}} \leqslant C\|u\|_{\mathscr{H}(\mathbf{m}, p, s)} .
$$

Наконец, равномерная ограниченность операторов $\mathscr{L}^{-1}(s, 1 / 2)$ видна из доказательства теоремы 1. Действительно, положим $\gamma_{s}=\left\|\mathscr{L}^{-1}(s, 1 / 2)\right\|$ и обозначим через $\gamma_{1}$ норму этого же оператора из $\left[\widetilde{\mathscr{H}}^{-\mathbf{m}}(\Omega), \widetilde{\mathscr{H}}^{-\mathbf{m}+1}(\Omega)\right]_{1 / 2,1}$ в 
$\mathscr{B}_{p}^{\mathbf{m}+1 / 2}(\Omega)$. Тогда при $0<s<1 / 2$

$$
\gamma_{s} \leqslant C^{\prime} \gamma_{0}^{1-2 s} \gamma_{1}^{2 s} \leqslant C^{\prime \prime}
$$

где постоянные $C^{\prime}$ и $C^{\prime \prime}$ не зависят от $s$ (см., например, [5], пп. 2.4 и 6.4). Равномерная ограниченность оператора $\mathscr{L}^{-1}(s, 1 / 2)$ при $0<s<1 / 2$ получается по дуальности.

Примеры, показывающие, что в общем случае нельзя $\delta$ заменить единицей (и даже фиксированным меньшим числом), известны. См. ссылки, например, в [11], [1], [29]. Там же указаны случаи, когда область $Q_{\delta}$ однозначной разрешимости можно, наоборот, существенно расширить.

Из теоремы 3 вытекает утверждение о регулярности решений:

Теорема 4. Пусть точки $\left(s_{1}, t_{1}\right)$ u $\left(s_{2}, t_{2}\right)$ принадлежат $Q_{\delta}$, причем пространства, отвечающие второй точке, содержсатся в соответствующих пространствах, отвечающих первой точке. Тогда если $\mathscr{L} u=f$ в первой точке, но $f$ «лучше» - лежит в пространстве, отвечающем второй точке, то это же верно для решения и.

\section{§5. Некоторые обобщения, варианты и следствия}

В ближайших двух пунктах мы обсуждаем возможности рассмотрения неоднородных условий Дирихле и Неймана. В случае липшицевой области мы должны указать определения по аналогии со случаем гладкой области и предположения, при которых готовы рассматривать такие условия. Для экономии места и простоты мы рассмотрим скалярное уравнение, перенесение сказанного ниже на системы не вызывает затруднений. Вместо $\mathbf{m}$ теперь будем писать $m$. Эти два пункта и п. 5.5 написаны несколько конспективно из-за недостатка места; подробности см. в [4].

5.1. Неоднородные условия Дирихле и наборы Уитни. Задачу с неоднородными условиями Дирихле поставим следующим образом. Заданы функции $u_{0} \in \mathscr{H}(m, p, s)$ и $f \in \mathscr{H}(-m, p, s)$; требуется найти такое решение $u$ уравнения $L u=f$ в первом из этих пространств, что $u-u_{0} \in \widetilde{\mathscr{H}}(m, p, s)$.

Определим $f_{0}=L u_{0} \in \widetilde{\mathscr{H}}(-m, p, s)$ тождеством

$$
\left(f_{0}, v\right)_{\Omega}=\Phi_{\tau}\left(u_{0}, v\right) \quad\left(v \in \mathscr{H}\left(m, p^{\prime},-s\right)\right),
$$

тем самым подчиняя $u_{0}$ однородным условиям Неймана. Теперь дело сводится к решению уравнения $L\left(u-u_{0}\right)=f-f_{0}$ с однородным условием Дирихле.

Здесь, однако, надо пояснить следующее. Так как $m-1 / 2+s$ нецелое, то $\widetilde{\mathscr{H}}(m, p, s)$ можно отождествить с подпространством $\stackrel{\circ}{H}_{p}^{m-1 / 2+1 / p+s}(\Omega)$ в $\mathscr{H}(m, p, s)$. Поэтому условие $u-u_{0} \in \widetilde{\mathscr{H}}(m, p, s)$ имеет смысл. Далее, из $(5.1)$ по аналогичной причине следует это же соотношение для $v \in \widetilde{\mathscr{H}}\left(m, p^{\prime},-s\right)$. Это позволяет рассматривать $f_{0}$ как элемент из $\mathscr{H}(-m, p, s)$, и тогда $f-f_{0}$ принадлежит этому же пространству. Поэтому корректно поставлена задача Дирихле для нахождения $u-u_{0}$.

Следствие 1. Если в точке $(s, t)$ однозначно разрешима задача Дирихле $c$ однородными граничными условиями, то это же верно для задачи Дирихле с неоднородными граничными условиями. 
Теперь мы сопоставим этот подход с постановкой неоднородных условий Дирихле в терминах наборов Уитни, в близкой ситуации введенных в [28].

В используемых ниже пространствах Бесова $B_{p}^{\sigma}(\Gamma)=B_{p, p}^{\sigma}(\Gamma), 0<\sigma<1$, $1<p<\infty$, норму можно определить равенством

$$
\|u\|_{B_{p}^{\sigma}(\Gamma)}^{p}=\|u\|_{L_{p}(\Gamma)}^{p}+\int_{\Gamma} \int_{\Gamma} \frac{|u(x)-u(y)|^{p}}{|x-y|^{n-1+\sigma p}} d S_{x} d S_{y} .
$$

Эту шкалу можно продолжить на значения $\sigma \in[-1,1]$, но дальше нельзя: инвариантные определения пространств $B_{p}^{\sigma}(\Gamma)$ на общей липшицевой поверхности есть только при $|\sigma| \leqslant 1$. След функции $u \in H_{p}^{\sigma+1 / p}(\Omega)$ на $Г$ принадлежит $B_{p}^{\sigma}(\Gamma)$, но, вообще говоря, только при $0<\sigma<1$ (см. [12], стр. 182, определения пространств Бесова в этой книге, [11] и указанные там ссылки). Однако при $\sigma>k+1 / p$ с натуральным $k$ следы имеют также производные до порядка $k$ включительно.

Пусть функция $u$ принадлежит $H_{p}^{\sigma+1 / p}(\Omega)$ с большим $\sigma$. Идея введения наборов Уитни состоит в рассмотрении (согласованного) набора граничных значений производных от $u$ в пространствах функций на границе малой гладкости. Ниже мы в основном следуем [1] и [14], где существенны связи с концепциями в [12] и [32].

Далее в этом пункте будем предполагать, что $q$ - целое неотрицательное число и $\sigma \in(q, q+1)$. Связь с использованными выше обозначениями выражается равенствами $q=m-1, \sigma=m-1 / 2+s=q+1 / 2+s$. В случае бигармонического уравнения $q=1$.

Обозначим через $\Pi_{p}^{\sigma}(\Gamma)$ пространство наборов $\dot{w}=\left\{w_{\alpha}\right\}_{|\alpha| \leqslant q}$ функций $w_{\alpha}(x)$ на $\Gamma$, принадлежащих $H_{p}^{1}(\Gamma)$ при $|\alpha|<q$ и $B_{p}^{\sigma-q}(\Gamma)$ при $|\alpha|=q$. Введем в $\Pi_{p}^{\sigma}(\Gamma)$ норму

$$
\|\dot{w}\|_{\Pi_{p}^{\sigma}(\Gamma)}=\sum_{|\alpha|<q}\left\|w_{\alpha}(x)\right\|_{H_{p}^{1}(\Gamma)}+\sum_{|\alpha|=q}\left\|w_{\alpha}(x)\right\|_{B_{p}^{\sigma-q}(\Gamma)} .
$$

Это, конечно, банахово пространство.

Пусть сначала $u(x)$ - функция из $C_{0}^{\infty}\left(\mathbb{R}^{n}\right)$. Рассмотрим набор $\dot{w}(x)=\left\{w_{\alpha}(x)\right\}$ следов $w_{\alpha}(x)$ на $\Gamma$ всевозможных производных $\partial^{\alpha} u(x)$ порядка $|\alpha| \leqslant q$. Заметим, что элементы $w_{\alpha}$ с $|\alpha|<q$ фактически принадлежат $H_{p}^{1}(\Gamma)$, так как их касательные производные принадлежат $L_{2}(\Gamma)$, и что выполнены условия согласованности: для всех возможных значений индексов

$$
\left[\nu_{j}(x) \partial_{k}-\nu_{k}(x) \partial_{j}\right] w_{\alpha}(x)=\nu_{j}(x) w_{\alpha+e_{k}}(x)-\nu_{k}(x) w_{\alpha+e_{j}}(x)
$$

при $|\alpha| \leqslant q-1$, где $\nu=\left(\nu_{1}, \ldots, \nu_{n}\right)$ - единичная внешняя нормаль и $e_{k}$ мультииндекс из нулей и единиц с единицей только на $k$-м месте. Это следует из того, что слева и справа записаны следы одних и тех же производных.

Такие наборы образуют линеал в $\Pi_{p}^{\sigma}(\Gamma)$. Замыкание этого линеала обозначим через $\mathrm{WA}_{p}^{\sigma}(\Gamma)$, а элементы $\dot{w}$ этого замыкания назовем наборами Уитни порядка $(\sigma, p)$ на Г. Другое, эквивалентное определение будет приведено ниже.

Пусть теперь $u(x)$ - функция из $H_{p}^{\sigma+1 / p}(\Omega)$. Тогда можно образовать набор $\left\{\partial^{\alpha} u(x)\right\}$ из значений ее производных порядка $|\alpha| \leqslant q$ на $\Gamma$, тоже имеющий конечную норму (5.3). Мы утверждаем, что это набор Уитни. Действительно, 
функцию $u(x)$ можно продолжить до функции из $H_{p}^{\sigma+1 / p}$ с компактным носителем [22] и там аппроксимировать бесконечно гладкими финитными функциями $u_{k}(x)$ в норме этого пространства. Тогда наборы Уитни $\left\{\partial^{\alpha} u_{k}(x)\right\}$ будут сходиться к набору $\left\{\partial^{\alpha} u(x)\right\}$ в пространстве $\Pi_{p}^{\sigma}(\Gamma)$. Предельным переходом получаются условия согласованности (5.4).

Следующее утверждение известно специалистам, см. [28] и [1], но проверено в литературе, по-видимому, только для $q=1$ - в работе [1].

Теорема 5. Пусть $\dot{w}=\left\{w_{\alpha}\right\} \in \Pi_{p}^{\sigma}(\Gamma), q<\sigma<q+1$, и для $w_{\alpha}$ выполнены условия согласованности (5.4). Тогда существует такая функиия $и \in$ $H_{p}^{\sigma+1 / p}(\Gamma)$, что следъ ее производнъх $\left.\partial^{\alpha} u(x)\right|_{\Gamma}$ образуют набор $\dot{w}$. Oператор $\mathscr{E}_{q}: \dot{w} \rightarrow u$ линеен, ограничен в указанных пространствах и зависит только orm $q$.

Это вытекает из нетривиальных результатов в [12] в главе VII. Но, чтобы воспользоваться ими, надо отождествить пространства только что указанных наборов с пространствами из [12]. Для $q=1$ это проделано в [1], см. там лемму 1.7. В общем случае мы используем леммы из [14], §7. Подробности см. в [4]. В [14] вместо $H_{p}^{\sigma+1 / p}(\Omega)$ рассматривается весовое пространство с целочисленным верхним индексом.

Из теоремы 5 вытекает, что определение пространства $\mathrm{WA}_{p}^{\sigma}(\Gamma)$, данное выше, равносильно следующему: это подпространство в $\Pi_{p}^{\sigma}(\Gamma)$, состоящее из наборов $\dot{w}=\left\{w_{\alpha}\right\}$, которые удовлетворяют условиям согласованности.

В [28] рассматривались только целочисленные $\sigma$, у нас они не возникают. В [1] $1<\sigma \leqslant 2$ и главное внимание уделено случаю $\sigma=2$, при нецелых $\sigma$ использовалась интерполяция. Случай нецелых $\sigma$ проще. См. [4].

Сказанное позволяет несколько углубить постановку задачи Дирихле с неоднородными граничными условиями следующим образом. Задается набор Уитни $\dot{w}(x)$ в пространстве $\mathrm{WA}_{p}^{m-1 / 2+s}(\Gamma)$, который должен быть набором из значений производных решения на границе. По нему строится функция $u_{0} \in \mathscr{H}(m, p, s)$, для которой этот набор является набором из значений ее производных на границе. Далее определяем решение как в начале настоящего пункта.

Смысл сказанного состоит в совместимости вариационного подхода с использованием наборов Уитни.

Рассмотрим теперь неоднородную задачу Дирихле в обычной форме (считая функции скалярными):

$$
L u=f \quad \text { в } \Omega, \quad \partial_{\nu}^{k} u=g_{k}(k=0, \ldots, m-1) \text { на } \Gamma .
$$

Здесь $\partial_{\nu}$ - производная по внешней нормали, $f \in \mathscr{H}(-m, p, s),(s, t) \in Q_{\delta}$. Нас интересует, когда эта задача имеет (одно и только одно) решение в $\mathscr{H}(m, p, s)$. Мы можем указать достаточные условия в следующей форме. Предположим, что существует набор Уитни $\left\{w_{\alpha}\right\}$ порядка $(m-1 / 2+s, p)$, порождающий эти производные по нормали. Тогда ситуация совпадает с только что рассмотренной и однозначная разрешимость имеет место. Поясним слово «порождающий». 
Производные по нормали определяются через элементы набора Уитни (производных некоторой функции) следующей известной формулой (см. [28]):

$$
\partial_{\nu}^{k} u(x)=\sum_{|\alpha|=k} \frac{k !}{\alpha !} \nu^{\alpha} w_{\alpha}
$$

где $\nu^{\alpha}=\nu_{1}^{\alpha_{1}} \cdots \nu_{n}^{\alpha_{n}}$. Функции $\nu_{j}$ в общем случае только ограничены и измеримы. Поэтому относительно функций $g_{k}$ можно утверждать лишь, что они принадлежат $L_{p}(\Gamma)$.

Имеются также формулы для восстановления набора $\left\{w_{\alpha}\right\}$ по этим нормальным производным (см. в [14] теорему 7.8). Таким образом, в том и только в том случае, если эти формулы приводят к набору Уитни порядка $(m-1 / 2+s, p)$, задача (5.5) удовлетворяет нашим предположениям, гарантирующим однозначную разрешимость. Ср. [28] и [14].

5.2. Неоднородные условия Неймана. Предположим сначала, что граница области является гладкой. Тогда можно написать полную формулу Грина с граничными членами. Пусть она имеет вид

$$
(f, u)_{\Omega}=\Phi_{\sigma}(u, v)+\sum_{j=0}^{m-1}\left(B_{m-1-j} u, \partial_{\nu}^{j} v\right)_{\Gamma} .
$$

Данными Неймана будем считать выражения $\left.B_{m-1-j} u\right|_{\Gamma}$. Таким образом, мы хотим рассмотреть задачу

$$
L u=f \text { в } \Omega, \quad B_{m-1-j} u=h_{m-j-1}(j=0, \ldots, m-1) \text { на } \Gamma
$$

уже в липшицевой области, снова считая, что $f \in \widetilde{\mathscr{H}}(-m, p, s)$ и $(s, t) \in Q_{\delta}$.

Подставим в (5.7) выражения (5.6) и перебросим негладкие коэффициенты на функции до запятой. Правая часть в $(5.7)$ примет вид $\sum\left(\widetilde{h}_{\alpha}, \partial^{\alpha} v\right)_{\Gamma}$. Мы можем гарантировать однозначную разрешимость задачи (5.8), если получился линейный непрерывный функиионал над $\mathrm{WA}_{p^{\prime}}^{m-1 / 2-s}(\Gamma)$ (см. [4]). Действительно, это тогда линейный непрерывный функционал над $\mathscr{H}\left(m, p^{\prime},-s\right)$, а это позволяет изменить правую часть уравнения $L u=f$ так, что граничные условия Неймана станут однородными. Ср. [30].

Конечно, значительно прозрачнее все это выглядит в случае уравнений и систем 2-го порядка. В этом случае легко указывается пространство для данных Неймана и всегда можно предполагать условия Неймана однородными, не ограничивая общности. См. [3].

5.3. Уравнения с младшими членами. Пусть $L$ содержит младшие члены. Если они записаны в дивергентной форме, то при выводе неравенства Гординга коэффициенты в них считают измеримыми ограниченными функциями, а в теоремах о регулярности достаточно подчинить их равномерному условию Липшица.

Следствие 2. Утверждение об однозначной разрешимости задач Дирихле u Неймана при наличии младших членов в L сохраняется, если $\operatorname{Re} \tau$ достаточно велико. При остальных $\tau$ задача фредгольмова в тех же самых пространствах с нулевым индексом. Сохраняется утверждение о гладкости. 
Поясним, что результат о гладкости в случае фредгольмовости получается перенесением младших членов в правую часть уравнения с учетом специфики определения вариационного решения.

5.4. Спектральные задачи. Мы можем рассмотреть спектральное уравнение $L u=\lambda u$ с однородными условиями Дирихле или Неймана. Сохраним те же предположения об операторе $L$, что и в неспектральных задачах. Предположим, что нуль не является собственным значением. Тогда из тех же соображений, что и в [3], получается

Следствие 3. Собственные и присоединенные функиии $u(x)$ спектральных задач Дирихле и Неймана принадлежст пересечению пространств, отвечающих точкам $(s, t) \in Q_{\delta}$.

В случае систем 2-го порядка получается аналогичный результат и для задачи со спектральным параметром в граничном условии, т. е. для собственных и корневых функций оператора, при $f=0$ переводящего данные Неймана в данные Дирихле.

5.5. Аналоги результатов для пространств Бесова. Все сказанное в $\S 4$ и предыдущих пунктах настоящего параграфа переносится на пространства Бесова вместо пространств потенциалов. Пусть снова выполнены условия (4.1). Одно из простейших определений пространства $B_{p}^{\sigma}=B_{p}^{\sigma}\left(\mathbb{R}^{n}\right)$, cp. [11], состоит в том, что при нецелом $\sigma>0$ оно совпадает с пространством Слободецкого $W_{p}^{\sigma}=W_{p}^{\sigma}\left(\mathbb{R}^{n}\right)$ с нормой

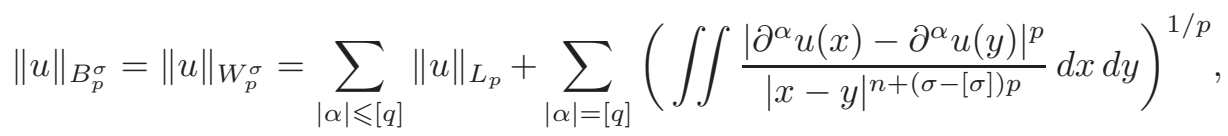

а при остальных $\sigma$ это лежащее в пространстве Шварца $\mathscr{S}^{\prime}=\mathscr{S}^{\prime}\left(\mathbb{R}^{n}\right)$ пространство с нормой

$$
\|u\|_{B_{p}^{\sigma}}=\left\|\Lambda^{\sigma-\sigma_{1}} u\right\|_{B_{p}^{\sigma_{1}}}
$$

где $\sigma_{1}$ нецелое положительное; при его изменении норма переходит в эквивалентную. Относительно других возможных определений этих пространств см., например, [19] или [26]. Для простоты и экономии места мы не будем рассматривать пространства Бесова с тремя индексами. Известно, что пространства $B_{p}^{\sigma}$ и $B_{p^{\prime}}^{-\sigma}$ взаимно сопряжены относительно продолжения скалярного произведения в $L_{2}$ на их прямое произведение. Пространство $B_{p}^{\sigma}(\Omega)$ вводится аналогично $H_{p}^{\sigma}$, т. е. как состоящее из сужений функций из $B_{p}^{\sigma}($ обобщенных при $\sigma<0)$ на $\Omega$. Универсальный оператор продолжения $\mathscr{E}$ из [22] обслуживает и эти пространства. Пространство $\widetilde{B}_{p}^{\sigma}(\Omega)$ вводится как подпространство в $B_{p}^{\sigma}$, состоящее из функций (обобщенных при $\sigma<0$ ) с носителями в $\bar{\Omega}$. При $\sigma_{1}<\sigma_{2}$ имеют место непрерывные вложения

$$
H_{p}^{\sigma_{2}} \subset B_{p}^{\sigma_{1}}, \quad B_{p}^{\sigma_{2}} \subset H_{p}^{\sigma_{1}}
$$

а при $p=2$

$$
H_{2}^{\sigma}=B_{2}^{\sigma}\left(=H^{\sigma}=W_{2}^{\sigma}\right) .
$$

Соотношения (5.11)-(5.12) наследуются пространствами $H_{p}^{\sigma}(\Omega)$ и $B_{p}^{\sigma}(\Omega), \widetilde{H}_{p}^{\sigma}(\Omega)$ и $\widetilde{B}_{p}^{\sigma}(\Omega)$. Пространства $B_{p}^{\sigma}(\Omega)$ и $\widetilde{B}_{p^{\prime}}^{-\sigma}(\Omega)$ оказываются взаимно сопряженными относительно формы (4.2). Короче говоря, картина для пространств Бесова 
аналогична картине для пространств потенциалов. Точно так же обстоит дело с комплексной интерполяцией (ср. [5], п. 6.4.5).

Далее для задач Дирихле и Неймана с однородными граничными условиями вводятся аналогичные пространствам (4.4) и (4.5) пространства $\mathscr{B}( \pm \mathbf{m}, s, p)$ и $\widetilde{\mathscr{B}}( \pm \mathbf{m}, s, p)$. В задаче Дирихле можно считать, что

$$
u \in \widetilde{\mathscr{B}}(\mathbf{m}, p, s), \quad v \in \widetilde{\mathscr{B}}\left(\mathbf{m}, p^{\prime},-s\right), \quad f \in \mathscr{B}(-\mathbf{m}, p, s),
$$

а в задаче Неймана - что

$$
u \in \mathscr{B}(\mathbf{m}, p, s), \quad v \in \mathscr{B}\left(\mathbf{m}, p^{\prime},-s\right), \quad f \in \widetilde{\mathscr{B}}(-\mathbf{m}, p, s) .
$$

Наши основные результаты в $\S 4$ дословно переносятся на пространства $\mathscr{B}$ и $\widetilde{\mathscr{B}}$ вместо $\mathscr{H}$ и $\widetilde{\mathscr{H}}$ вместе с доказательствами:

Теорема $\mathbf{3}^{\prime}$. Теорема 3 остается в силе, если заменить пространства $\mathscr{H}$ и $\widetilde{\mathscr{H}}$ пространствами $\mathscr{B}$ и $\widetilde{\mathscr{B}}$.

Теорема $4^{\prime}$. Теорема 4 остается в силе, если заменить пространства $\mathscr{H}$ и $\widetilde{\mathscr{H}}$ пространствами $\mathscr{B}$ и $\widetilde{\mathscr{B}}$.

В силу теоремы 1 из главы $\mathrm{V}$ в [12], при $0<\sigma<1$ оператор перехода к следу функции из $B_{p}^{\sigma+1 / p}(\Omega)$ действует ограниченным образом из этого пространства в то же, что и в случае пространства $H_{p}^{\sigma+1 / p}(\Omega)$, пространство $B_{p}^{\sigma}(\Gamma)$, а правый обратный оператор действует ограниченным образом из последнего пространства и в $B_{p}^{\sigma+1 / p}(\Omega)$, так что он фактически приводит в пересечение $B_{p}^{\sigma+1 / p}(\Omega) \cap H_{p}^{\sigma+1 / p}(\Omega)$.

Переходя к задачам Дирихле и Неймана с неоднородными граничными условиями, можно повторить сказанное в пn. 5.1 и 5.2 с пространствами Бесова вместо пространств потенциалов. При этом наше рассмотрение этих задач снова совместимо с использованием наборов Уитни. Их определения остаются теми же, что и в п. 5.1. В частности, верна

Теорема $5^{\prime}$. Пусть $\dot{w}=\left\{w_{\alpha}\right\} \in \Pi_{p}^{\sigma}(\Gamma), \sigma \in(q, q+1)$, u nусть выполнены условия согласованности (5.4). Тогда существует такая функиия $и \in$ $B_{p}^{\sigma+1 / p}(\Gamma)$, что следы ее производных $\left.\partial^{\alpha} u(x)\right|_{\Gamma}$ образуют набор $\dot{w}$. Oператор $\mathscr{E}_{q}: \dot{w} \rightarrow$ линеен, ограничен в указанных пространствах и зависит только om $q$.

Более того, этот оператор совпадает с оператором $\mathscr{E}_{q}$ из теоремъ 5, так что он бактически действует из $\mathrm{WA}_{p}^{\sigma}(\Gamma)$ в пересечение $B_{p}^{\sigma+1 / p}(\Omega) \cap H_{p}^{\sigma+1 / p}(\Omega)$.

Все это снова выводится из результатов в [12] (главы VI-VII), а при $q=1$ проверено в [1].

Рассмотрим задачу Дирихле для системы $L u=f$, где $f \in \mathscr{H}(-m, p, s) \cap$ $\mathscr{B}(-m, p, s)$. Предположим, что $(s, t) \in Q_{\delta}$, где $\delta$ в формулировках теорем 3 и $3^{\prime}$ будем считать общим, и пусть наборы Уитни для данных Дирихле содержатся в $\mathrm{WA}_{p}^{m_{j}-1 / 2+s}(\Gamma)$. Вектор-функция $u_{0}$, которая строится по этим наборам Уитни, согласно теореме $5^{\prime}$ принадлежит пересечению $\mathscr{H}(\mathbf{m}, p, s) \cap \mathscr{B}(\mathbf{m}, p, s)$.

Следствие 4. При этих предположениях для решения задачи Дирихле имеeм $u-u_{0} \in \widetilde{\mathscr{H}}(\mathbf{m}, p, s) \cap \widetilde{\mathscr{B}}(\mathbf{m}, p, s)$. 


\section{§6. Замечания о литературе}

Начало «невариационному направлению» положили работы Кальдерона и Дальберга (1977). Этот подход теперь кратко можно охарактеризовать как основанный на использовании поверхностных потенциалов и исследовании их сходимости в некасательных конусах при приближении к Г, контролируемой в терминах максимальных функций (максимумов модуля по этим конусам). Используются глубокая теорема Койфмана-Макинтоша-Мейера об ограниченности сингулярных интегральных операторов в $L_{2}(\Gamma)$ и $L_{p}(\Gamma)(1988)$ и многие другие тонкие средства из анализа. Мы привели довольно большую литературу по работам этого направления об уравнениях и системах 2-го порядка в [3], и ее нет смысла здесь повторять. Добавим только, что решения в двух шкалах пространств, потенциалов и Бесова, в случае задачи Дирихле для уравнений Лапласа и Пуассона рассмотрены в [11]; развитие этих результатов см. в [10], [33] и [15].

Остановимся кратко на работах по уравнениям и системам высших порядков. Здесь исследования главным образом были направлены на изучение задачи Дирихле. Скрупулезное исследование этой задачи в двух шкалах для бигармонического уравнения $\Delta^{2} u=0$ проведено в [1], см. также [8]. Задача Дирихле для полигармонического уравнения $\Delta^{m} u=0$ рассмотрена в [28]. Задача Неймана для бигармонического уравнения исследована в [30].

Задача Дирихле для однородных относительно дифференцирования сильно эллиптических систем высокого порядка с постоянными вещественными коэффициентами и симметричной главной частью рассматривалась в [29], [24], [7]. Наконец, задача Дирихле для общей сильно эллиптической системы с переменными коэффициентами и старшей частью, однородной относительно дифференцирования, обстоятельно изучена в препринте [14] в весовых пространствах потенциалов. Решения и правые части принадлежат пространствам с целочисленными верхними индексами и произвольным нижним индексом $p \in(1, \infty)$. Вес $\rho(x)$ равен некоторой степени регуляризованного расстояния от точки $x \in \Omega$ до границы и характеризует гладкость функции. На липшицеву постоянную наложено некоторое ограничение сверху интегрального характера.

Без сомнения, в этих работах трактовка граничных условий является более глубокой, чем при нашем подходе. Нам удалось обойтись без сделанных в [29], [7], [24] дополнительных предположений о коэффициентах и без дополнительных предположений о нормали к Г, сделанных в [14], при этом для задачи Неймана удалось получить не менее общие результаты, чем для задачи Дирихле. Однако нам пришлось предположить выполненным условие (2.11).

В отличие от только что упомянутых работ у нас совсем не используются поверхностные потенциалы. По-видимому, наоборот, из наших результатов могут быть получены некоторые следствия для уравнений с этими потенциалами.

Мы не затрагивали здесь многочисленные исследования граничных задач в областях с особенностями границы специального вида (В. А. Кондратьев, В. А. Козлов, В. Г. Мазья, Ю. Россман, С. А. Назаров, Б. А. Пламеневский и др.), а также более специальные результаты для задач в липшицевых областях. См. ссылки в [14]. 


\section{ЛитеРАТУРА}

[1] V. Adolfsson, J. Pipher, The inhomogeneous Dirichlet problem for $\Delta^{2}$ in Lipschitz domains, J. Funct. Anal., 159 (1998), 137-190.

[2] М. С. Агранович, Спектральные задачи для силъно эллиптических систем второго порядка в областях с гладкой и негладкой гранищей, УМН, 57:5 (2002), 3-79.

[3] М. С. Агранович, Регулярность вариачионных решений линейных граничных задач в липшищевых областях, Функц. анализ и его прил., 40:4 (2006), 83-103.

[4] M. S. Agranovich, Remarks on spaces of potentials and Besov spaces in a Lipschitz domain and of Whitney arrays on its boundary, Russian J. Math. Phys., 16:2 (2008) (в печати).

[5] Й. Берг, Й. Лёфстрём, Интерполячионные пространства. Введение, Мир, М., 1980 .

[6] M. Costabel, Boundary integral operators on Lipschitz domains: elementary results, SIAM J. Math. Anal., 19:3 (1988), 613-626.

[7] B. E. J. Dahlberg, C. E. Kenig, J. Pipher, G. C. Verchota, Area integral estimates for higher order elliptic equations and systems, Ann. Inst. Fourier (Grenoble), 47 (1997), 1425-1461.

[8] B. E. J. Dahlberg, C. E. Kenig, G. C. Verchota, The Dirichlet problem for the biharmonic equation in a Lipschitz domain, Ann. Inst. Fourier (Grenoble), 36:3 (1986), $109-135$.

[9] А. Л. Гольденвейзер, В. Б. Лидский, П. Е. Товстик, Свободные колебания тонких упругих оболочек, Наука, 1979.

[10] E. Fabes, O. Mendes, M. Mitrea, Boundary layers on Sobolev-Besov spaces and Poisson's equation for the Laplacian in Lipschitz domains, J. Funct. Anal., 159 (1998), 323-368.

[11] D. Jerison, C. E. Kenig, The inhomogeneous Dirichlet problem in Lipschitz domains, J. Funct. Anal., 130 (1995), 164-219.

[12] A. Jonsson, H. Wallin, Function Spaces on Subsets of $\mathbb{R}^{n}$, Math. Rep., vol. 2, Harwood Academic Publ., 1984.

[13] P. D. Lax, N. Milgram, Parabolic equations, in: Contributions to the Theory of Partial Differential Equations, Ann. Math. Stud., vol. 33, 1954, 167-190.

[14] V. Maz'ya, M. Mitrea, T. Shaposhnikova, The Dirichlet problem in Lipschitz domains for higher order elliptic systems with rough coefficients, http://arxiv.org/abs/math/ 0701898, Jan. 2007.

[15] M. Mitrea, M. Taylor, Potential theory on Lipschitz domains in Riemannian manifolds: Sobolev-Besov space results and the Poisson problem, J. Funct. Anal., 176 (2000), 1-79.

[16] С. А. Назаров, Полиномиальное свойство эллиптических краевых задач и алгебраическое описание их атрибутов, УМН, 54:5 (1999), 77-142.

[17] J. Nečas, Les métodes directes en théorie des équations elliptiques, Masson, Academia, Paris-Prague, 1967.

[18] L. Nirenberg, Remarks on strongly elliptic partial differential equations, Comm. Pure Appl. Math., 8 (1955), 649-675.

[19] J. Peetre, New Thoughts on Besov Spaces, Duke Univ. Math. Series, vol. 1, Durham, N.C., 1976.

[20] J. Pipher, G. Verchota, Dilation invariant estimates and the boundary Gärding inequality for higher order elliptic operators, Ann. of Math., 142 (1995), 1-38.

[21] Р. Рокафеллар, Выпуклый анализ, Мир, М., 1973.

[22] V. S. Rychkov, On restrictions and extensions of the Besov and Triebel-Lizorkin spaces with respect to Lipschitz domains, J. London Math. Soc. (2), 60 (1999), 237-257. 
[23] J. Savaré, Regularity results for elliptic equations in Lipschitz domains., J. Funct. Anal., 152 (1998), 176-201.

[24] Z. Shen, Necessary and sufficient conditions for the solvability of the $L_{p}$ Dirichlet problem on Lipschitz domains, Math. Ann., 336 (2006), 697-795.

[25] И. Я. Шнейберг, Спектральные свойства линейных операторов в интерполяиионных семействах банаховых пространств, Матем. исслед., 9:2 (1974), 214-227.

[26] Х. Трибель, Теория интерполячии, функциональные пространства, дифберенииальные операторы, Мир, М., 1980.

[27] H. Triebel, Function spaces in Lipschitz domains and on Lipschitz manifolds. Characteristic functions as pointwise multipliers, Rev. Mat. Comput., 15 (2002), 475-524.

[28] G. Verchota, The Dirichlet problem for polyharmonic equation in Lipschitz domains, Indiana Univ. Math. J., 39 (1990), 671-702.

[29] G. Verchota, Potentials for the Dirichlet problem in Lipschitz domains., in: Potential Theory-ICPT 94, Valter de Gruyter, Berlin, 1996, 168-187.

[30] G. Verchota, The biharmonic Neumann problem in Lipschitz domains, Acta Math., 194 (2005), 217-279.

[31] М. И. Вишик, О сильно эллиптических системах дифференииальных уравнений, Матем. сб., 29 (71) (1951), 615-676.

[32] H. Whitney, Analytic extensions of differentiable functions defined in closed sets, Trans. Amer. Math. Soc., 36 (1934), 63-69.

[33] D. Zanger, The inhomogeneous Neumann problem in Lipschitz domains, Comm. Partial Differential Equations, 25:9-10 (2000), 1171-1808.

Московский институт электроники и математики

Поступило в редакцию

e-mail: magran@orc.ru

1 мая 2007 г. 